\section{G335(P) FEVER AFTER MENINGOCOCCAL B IMMUNISATION: A CASE SERIES}

${ }^{1} \mathrm{G}$ Campbell, ${ }^{2} \mathrm{R}$ Bland, 'S Hendry. 'Emergency Department, Royal Hospital for Children, Glasgow, UK; ${ }^{2}$ Paediatric Department, Royal Hospital for Children, Glasgow, UK

10.1136/archdischild-2018-repch.325

Objective To document the clinical features and management of infants presenting with fever after their first meningococcal $B$ vaccination, and develop guidance for clinicians.

Design A prospective case series over a 12 month period.

Setting The Royal Hospital for Children Glasgow, a tertiary paediatric hospital.

Patients Infants $\leq 3$ months of age who had received their first set of immunisations (including Bexsero) within the preceding 72 hours and reported fever.

Results Ninety two infants met the inclusion criteria, accounting for $0.78 \%$ of the local vaccinated population. The most commonly described features were poor feeding, sleepiness and irritability. Sixty six patients $(72 \%)$ were admitted to hospital. Median CRP was $12 \mathrm{mg} / \mathrm{L}$ and median WCC was $16 \times$ $10^{9} /$ L.Fifteen patients $(16 \%)$ had a lumbar puncture and were commenced on antibiotics. There was one confirmed bacterial infection in an infant who had presented with fever starting 54 hours after immunisation. All other microbiology samples were negative. There were no cases of missed serious bacterial infection (SBI) in those patients observed or discharged.

Conclusion Post-immunisation fever is a common presenting problem to the paediatric emergency department. Based on our findings, routine investigations are not warranted if the infant appears well on examination. Where other common side effects are present and there is clinical concern, a period of observation may be a reasonable option. We suggest selective use of investigations, especially inflammatory markers, that are unlikely to discriminate between serious bacterial infection and post-immunisation response. We advocate extra caution in infants presenting with fever more than 48 hours after immunisation.

\section{G336(P) THE IMPACT OF A YOUTH VIOLENCE INTERVENTION PROGRAMME ON REATTENDANCE RATES AND YOUNG PEOPLE'S WELLBEING}

${ }^{1} \mathrm{C}$ Travers, ${ }^{2} \mathrm{G}$ Hann. ${ }^{1}$ Paediatrics and Child Health, University College London, London, UK; ${ }^{2}$ Paediatric Emergency Department, North Middlesex University Hospital, London, UK

\subsection{6/archdischild-2018-rcpch.326}

Introduction Tackling gang violence is a significant challenge. With knife crime and serious youth violence on the rise, the Government has looked towards alternatives to punitive measures. Hospital-based youth violence intervention programmes (YVIP) are one alternative that aim to reduce risk and increase protective factors for gang-related youth violence. Users views of YVIPs have yet to be evaluated in the UK.

Aims We had 2 main aims:

- to compared reattendance rates to $\mathrm{A} \& \mathrm{E}$ of those who engaged with the YVIP and those that refused engagement

- to evaluate the YVIP by examining exit surveys of the young people who had completed the programme

Methods The reattendance rates were analysed for those attending for gang violence who either fully engaged, partially engaged or refused to engage with the programme. The exit survey responses of those patients who had fully engaged were evaluated. The exit survey contained statements that the completed service users responded to based on how strongly they agreed/disagreed with statements. This allowed self-assessment about whether the YVIP had any impact on their lifestyle.

Results Since its inception in October 2014, the YVIP had received 465 referrals. Only 18\% (85 patients) engaged with the service. At the time of study only 15 services users fully engaged and completed the programme. Those patients who had fully engaged had lower reattendance rates $(6.7 \%)$ than those patients who engaged and dropped out (19\%) or those who had refused to engage (26.7\%).

Every completed service user felt that the YVIP had provided helpful support. $33.3 \%(5 / 15)$ strongly agreed that they were much happier since joining the programme. $33.3 \%(5 / 15)$ agreed that they were happier and $33.3 \%(5 / 15)$ were neutral. $73.3 \%(5 / 15)$ felt they were less likely to reattend A and E due to youth violence, whilst $26.7 \%$ (4/15) were neutral. $100 \%(15 / 15)$ strongly agreed that it is a good idea to have a youth support service in the hospital.

Conclusions Although it is hard to engage young people who have been involved in gang violence, there is evidence of the usefulness of YVIPs on both reattendance rates and the well being of those who engage.

\section{G337(P) GENDER, ETHNICITY AND SOCIAL DISADVANTAGE PATTERNS IN ED ATTENDANCE - NOT SUCH A SIMPLE STORY}

${ }^{1} \mathrm{R}$ Choudhury, ${ }^{2} \mathrm{~L}$ Ahmed, ${ }^{2,3} \mathrm{G}$ Oligbu, ${ }^{1,2} \mathrm{M}$ Blair. ${ }^{1}$ Faculty of Medicine, Imperial College, London, UK; ${ }^{2}$ River Island Academic Centre for Paediatrics and Child Health, Northwick Park Hospital, London, UK; ${ }^{3}$ Paediatric Infectious Disease Research Group, St George's University of London, UK

\subsection{6/archdischild-2018-rcpch.327}

Background There are well established gender, ethnicity and socio-economic differences in the incidence and prevalence of epilepsy, autism and allergy sensitisation. ${ }^{1,2,3,4}$ Less is known about these differences within an ED setting.

Simons et al. noted a gender disparity with more boys presenting to ED with sickness, head injury and falls than girls, ${ }^{5}$ but this was not statistically significant.

Aim To establish the role of gender, ethnicity, and social deprivation as factors associated with presentation to ED in children under 5 .

Methods Study design: A retrospective study was conducted on a routine operational data set of basic demographic data collected from a large district general hospital.

Sample: Paediatric ED data 01/04/2015-31/03/2016 for children below 5 years. Levels of deprivation: 2015 Index of Multiple Deprivation. ${ }^{6}$ Office for National Statistics (ONS) mid-year data used for denominator.

Analysis: Descriptive analysis. Rate ratios were calculated for each age. Deciles were obtained for each postcode using 2015 Index of Multiple Deprivation data.

Results Of the 19267 under 16 year-olds paediatric attendances, $10925(56.7 \%)$ were less than 5 years of age. Of these, 6227 (57\%) were male; M:F ratio in ED 1.33 cf. 1.05 local population. The proportion of the population attending ED decreased with age. Presentation rate was consistently higher in males than in females, but the gender gap decreased with age. 
Ethnicity was recorded for $89.9 \%$ of attendances.

The gender difference in presentations was greatest for the black population (622 males vs 392 females, M:F ratio 1.59 cf. 1.01 population).

At extremes of IMD, the rates of presentation were lowest (4.8\% decile $1,7.3 \%$ decile 10$)$ and gender gap is least pronounced (male:female rate ratio 1.13 decile 1, 1.26 decile 10, greatest 1.39 decile 4 .

Conclusions and clinical implications More males attend ED than females in all ages and ethnic groups, with greatest gender disparity in the black population. In addition, it appears that the least and most deprived are least likely to present to ED. This study emphasises the utility of operational data for epidemiological research at local level and supports the need to further explore clinical and sociological reasons for differences in gender related attendances.

\section{REFERENCES}

1. Levine R, Kilbourne B, Rust G, Langston M, Husaini B, Gittner L, Sanderson M, Hennekens C. Social determinants and the classification of disease: Descriptive epiidemiology of selected socially mediated disease constellations. PLoS One 2014;9(11).

2. Retico AG Alessia, Tancredi R, Cosenza A, Apicella F, Narzisi A, Biagi L, Tosetti $M$, Muratori F, Calderoni $S$. The effect of gender on the neuroanatomy of children with autism spectrum disorders: A support vector machine case-control study. Mol Autism 2016;7(5).

3. Pickrell W, Lacey A, Bodger O, Demmler J, Thomas R, Lyons R, Smith P, Rees M, Kerr M. Epilepsy and deprivation, a data linkage study. Epilepsia 2015;56 (4):585-91.

4. Joseph C, Zoratti E, Ownby D, Havstad D, Nicholas C, Nageotte C, Misiak R Enbeg, Ezell $R$, Johnson $C$. Exploring racial differences in IgE- mediated food allergy in the WHEALS birth cohort. Ann Allergy Asthma Immunol 2016;116 (3):219- 224.

5. Simons J. Young children in A\&E: A local review. Paediatric Nursing 1999;24(7).

6. Department for Communities and Local Government. The English indices of deprivation $2015 ; 2015$

\section{G338(P) IMPROVING THE EMERGENCY DEPARTMENT EXPERIENCE FOR CHILDREN WITH AUTISM, AND THEIR FAMILIES}

K Taylor, E Cadman, S Burkitt, A Langseth. Paediatric Emergency Department, St Mary's Hospital, London, UK

\subsection{6/archdischild-2018-rcpch.328}

Aims The Emergency Department (ED) can be extremely stressful for children with autism spectrum disorders (ASD). We aim to improve the standard of care provided to children with ASD and their families in the ED by introducing measures to reduce anxiety and stress, whilst educating staff and improving staff confidence in managing ASD patients.

Methods We assembled a multi-disciplinary team to brainstorm ideas that would improve patient care and decided to introduce a Patient Passport and electronic Communication Needs Alert. We distributed a questionnaire to parents and staff over a 6 week period to explore staff confidence in looking after children with ASD and parent satisfaction with current/previous care experiences. We also explored whether staff and parents would support our proposed interventions, and asked for suggestions of other potential ideas to improve care.

Results We surveyed 25 Paediatric ED staff and 9 parents who presented with their children during the 6 week period. Staff

- $84 \%$ had no specific training in managing ASD patients

- $76 \%$ felt they would benefit from additional teaching
- Only 32\% felt 'very confident' or 'quite confident' in managing ASD patients

- $72 \%$ felt a Patient Passport would be useful

- $64 \%$ felt an electronic Communication Alert would be useful

Parents

- $89 \%$ would want to use a Patient Passport

- $89 \%$ want to be asked at triage about their child's communications needs

- Only 22\% have been asked about their child's communications needs on every occasion they have attended ED

Other suggestions included sensory toys, ear defenders and communication aids.

\section{Conclusions}

- On average at least 1 patient with ASD attends ED per week, so interventions are likely to be worthwhile

- Staff would benefit from additional training in autism management

- Staff and families strongly support introducing both a Patient Passport and Communication Alert

Next steps:

- Roll out Passport and Communication Alert for use in paediatric ED

- Liaise with Community Paediatric teams to distribute Passport further

- Teaching for staff on managing ASD patients

- $(\mathrm{P}) \mathrm{s}$ inviting parents to highlight their child's communication needs at triage

- Seek hospital charity funding for sensory equipment to use in ED such as ear defenders, PECS cards and social stories.

\section{G339(P) IS BLOOD CULTURE A USEFUL INVESTIGATION IN CHILDREN ADMITTED TO HOSPITAL WITH COMMUNITY- ACQUIRED PNEUMONIA?}

JM Adamson. Paediatric Emergency Department, Leicester Royal Infirmary, Leicester, UK, Paediatric Emergency Department, Children's University Hospital Temple Street, Dublin, Ireland

\subsection{6/archdischild-2018-rcpch.329}

Aims Several papers in recent years have challenged the utility of blood cultures in children presenting with community acquired pneumonia. We aim to determine the usefulness of blood cultures taken at the time of initial presentation in the subsequent management of children with lower respiratory tract infections in our institution.

Methods From a database of all blood cultures taken in the Paediatric Emergency Department (PED) and Children's Assessment Unit (CAU), we identified a cohort of children who had been admitted to hospital and discharged with a diagnosis of lower respiratory tract infections who had undergone a blood culture test at the time of initial assessment. We retrospectively reviewed the blood culture results, respiratory microbiology (if obtained) and discharge letters of all children (aged 016) who had blood cultures sent in January and May 2016 from ED or CAU and were subsequently discharged with 'lower respiratory tract infection' as their main diagnosis.

Results 105 patients were identified. The dataset was complete as all records were available via the hospital ICE system. Of 